\title{
Does feet position alter triceps surae EMG record during heel-raise exercises in leg press machine?
}

\author{
Reginaldo S. Pereira', Jônatas B. Azevedo ${ }^{2 *}$, Fabiano Politti2*, Marcos R. R. Paunksnis', Alexandre L. Evangelista ${ }^{3}$, \\ Cauê V. La Scala Teixeira ${ }^{4,5}$, Andrey J. Serra6 ${ }^{6}$ Angelica C. Alonso', Rafael M. Pitta', Aylton Figueira Júnior', \\ Victor M. Reis', Danilo S. Bocalini
}

\begin{abstract}
Background: muscle activation measured by electromyography (EMG) provides additional insight into functional differences between movements and muscle involvement. Objective: to evaluate the EMG of triceps surae during heel-raise exercise in healthy subjects performed at leg press machine with different feet positions. Methods: ten trained healthy male adults aged between 20 and 30 years voluntarily took part in the study. After biometric analyses the EMG signals were obtained using a 8-channel telemeterized surface EMG system (EMG System do Brazil, Brazil Ltda) (amplifier gain: 1000x, common rejection mode ratio $>100 \mathrm{~dB}$, band pass filter: 20 to $500 \mathrm{~Hz}$ ). All data was acquired and processed using a 16-bit analog to digital converter, with a sampling frequency of $2 \mathrm{kHz}$ on the soleus (Sol), medial (GM) and lateral (GL) gastrocnemius muscles in both legs, in accordance with the recommendations of SENIAN. The root mean square (RMS) of the EMG amplitude was calculated to evaluate muscle activity of the three muscles. After being properly prepared for eletromyography procedures, all subjects were instructed to perform 3 sets of 5 repetitions during heel-raise exercise using the maximal load that enabled 10 repetitions on leg press $45^{\circ}$ machine, each set being performed with one of the following feet positions: neutral ( 0 - $)$, internal and external rotation (both with $45^{\circ}$ from neutral position). The tests were sequential and applied a 5-minute rest interval between sets. The order of the tests was randomized. Results: thought had been found interaction $(F=0.27, P=0.75)$ on RMS parameters and feet position, the values of Sol muscle were significantly ( $F=17.86, P=0.003)$ lower compared with $G L$ and $G M$ muscles independently of feet position. Conclusion: The change in the feet position during the heel-rise exercise performed in the leg press does not influence the activation of the triceps surae, and the soleus is less activated than the gastrocnemius in that exercise.
\end{abstract}

Keywords: Leg Press, Feet Position, Strength Training, Root Mean Square, Muscle Activity.

\section{INTRODUCTION}

The triceps surae muscle (TS) is composed of three muscles: lateral and medial gastrocnemius (bi-articular) and soleus

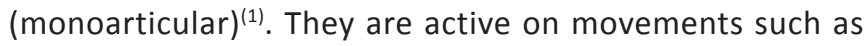
ankle plantar flexions and are also recruited during activities like walking, running, jumping, among others. Together, these muscles have had been considered as synergistic muscles in which different structural and physiological properties are combined in a unique mode, in order to supply movement demands $s^{(2)}$.

The TS muscle are attach to the calcaneo tendon, and during maximal plantar flexion efforts, all three muscles are contributing to the torque output and to the amount of tendon elongation ${ }^{(3,4)}$. The gastrocnemius (lateral and media) muscles across the knee and ankle joints and therefore, by varying the knee angle, the lengths of the medial and lateral gastrocnemius are altered while the length of the soleus muscle remains constant. In this way, its possible consider that myoletric activity of the muscle under investigation during different muscle action may be influenced by the joint angle or in different feet position.

Athletes and physical activity practitioners are concerned in training these muscles ${ }^{(5-7)}$. Muscle activation measuring by electromyography (EMG) provides additional insight into functional differences between muscles. Specifically, to the TS, several studies founded differences in activation during many modalities of exercise, such as cycling ${ }^{(8)}$, running $^{(9)}$ and resistance training ${ }^{(10)}$.

Presently, the exercise used to TS on strength training are often observed performing heel raises with foot in three different positions due to rationale that altering foot positions assumes that the different feet positions will prompt different muscle activation on TS muscle, however, to the best of our knowledge there are few studies ${ }^{(10)}$ that address this point on literature. In this way, considering that EMG is an efficient manner to provide objective rationale on which practitioners can base exercise-mode and intensity decisions the aim of 
this study was to evaluate the EMG of TS during heel-raise exercise in healthy subjects exercising at leg press machine with different feet positions.

\section{METHODS}

\section{Subjects}

A total of 10 trained healthy male adults aged between 20 and 30 years voluntarily took part in the study. The following criteria of exclusion were used: participation in current or previous regular exercise training in the last 6 months; recent hospitalization; symptomatic cardiometabolic or respiratory disease; cognitive impairment or progressive and debilitating conditions; marked obesity with inability to exercise; and recent bone fractures or any other medical contraindications to training. As inclusion criteria, men were aged 20 years or older and more than 6 moths of experience on strength training. The study was approved by the Institution Ethics Committee (2.100.780/2017) and all procedures followed the principles of the Declaration of Helsinki. The consent document was signed for publication of patient data.

\section{Anthropometrics parameters}

Height was measured to the nearest $0.1 \mathrm{~cm}$ using a Cardiomed stadiometer (Cardiomed Inc., WCS model, Curitiba, Brazil). Body mass was measured to the nearest $0.1 \mathrm{~kg}$ using a Filizola scale (Filizola Inc., Personal Line 150 model; São Paulo, Brazil). BMI ( $\left.\mathrm{kg} / \mathrm{m}^{2}\right)$ was calculated as follows: $\mathrm{BMI}=$ body mass / height / height $¥ 10000$. All procedures were carried out by the same researcher.

\section{Electromygraphy analyses}

EMG signals were obtained using a 8-channel telemeterized surface EMG system (EMG System do Brazil, Brazil Ltda) (amplifier gain: 1000x, common rejection mode ratio $>100 \mathrm{~dB}$, band pass filter: 20 to $500 \mathrm{~Hz}$ ). All data was acquired and processed using a 16-bit analog to digital converter, with a sampling frequency of $2 \mathrm{kHz}$. Disposable circular electrodes (Ag/AgCl, Medical Trace ${ }^{\circledR}$, Kendall Healthcare) with $10 \mathrm{~mm}$ diameter were attached with a center-to-center distance of $20 \mathrm{~mm}$ on the soleus (Sol), medial (GM) and lateral (GL) gastrocnemius muscles in both legs, in accordance with the recommendations of SENIAN ${ }^{(11)}$. The skin was cleaned with $70 \%$ alcohol before the electrodes were attached. The root mean square (RMS) of the EMG amplitude was calculated to evaluate muscle activity (Sol, GM and GL) after the tasks. The mean of the RMS obtained in three tests was used to compare the influence of feet position on the activity of each muscle that was studied. All EMG signals were processed using specific routines in the Matlab program, version R2016b (MathWorks Inc., Natick, Massachusetts, United States).

After being properly prepared for eletromyography procedures, all subjects were instructed to perform 3 sets of
5 repetitions during heel-raise exercise using the maximal load that enabled 10 repetitions on leg press $45^{\circ}$ machine, each set being performed with one of the following feet positions: neutral ( 0 - ) , internal and external rotation (both with $45^{\circ}$ from neutral position). The tests were sequential and applied a 5-minute rest interval between sets. The order of the tests was randomized.

\section{Statistical Analysis}

Data normality was confimred with Shapiro-Wilk test. A two-way analysis of variance (ANOVA) design was used to analyze the influence of feet positions considering with factors: positions of feet (neutral vs lateral rotation vs medial rotation) and muscles (Sol vs GM vs GL) with Tukey HSD post-hoc test. Partial eta squared $\left(\eta_{p}{ }^{2}\right)$ were used to calculate the effect size ${ }^{(12)}$. The interpretation was based on the values established by Cohen ${ }^{(12)}$ : small effect $\left(\eta_{p}^{2}=0.01\right)$; moderate effect (approximately $\eta_{p}{ }^{2}=0.06$ ); and large effect (greater than $\left.\eta_{p}^{2}=0.14\right)$.

\section{RESULTS}

At table 1 is showed the anthropometrics profile. Figure 1 shows the RMS parameters of Sol, GL and GM muscle of both body sides.

It was not found a significant interaction on feet positions and muscle $\left(F=0.09, P=1.00 ; \eta_{p}^{2}=0.005\right)$ as well as on feet position $\left(F=0.27, P=0.75 ; \eta_{p}^{2}=0.003\right)$. However, the mioletric activity was different between muscle ( $F=17.86$,

Table 1. Characteristics of the sample

\begin{tabular}{ccc}
\hline Parameters & Mean \pm SD & $95 \%$ of $\mathrm{Cl}$ \\
\hline Body mass $(\mathrm{kg})$ & $77.78 \pm 12.11$ & $69.08-84.47$ \\
Height $(\mathrm{m})$ & $1.72 \pm 0.12$ & $1.69-1.73$ \\
BMI $\left(\mathrm{kg} / \mathrm{m}^{2}\right)$ & $25.67 \pm 0.84$ & $23.80-27.53$ \\
\hline
\end{tabular}

Note: Values are presented as mean \pm standard deviation. BMI: body mass index. $\mathrm{Cl}$ : confidence interval.

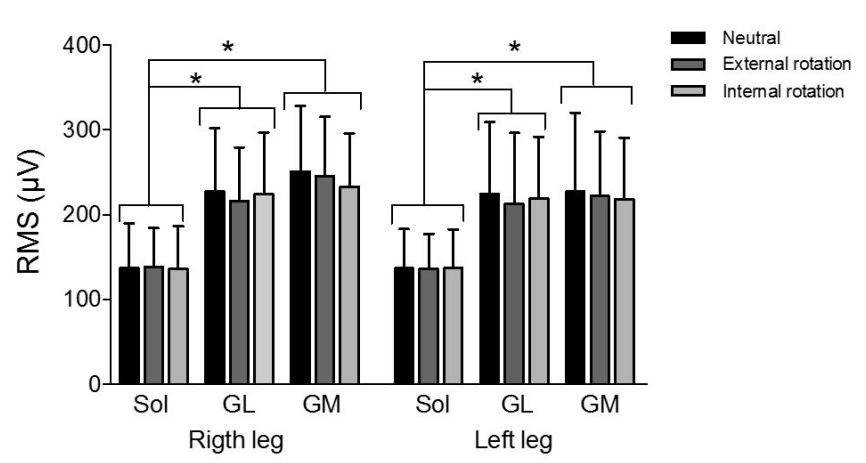

Figure 1. Mean and standard deviation of the RMS recorded with the feet in neutral, external and internal rotation. Sol: soleus muscle. GL: lateral gastrocnemius muscle. GM: medial gastrocnemius muscle. * Significant difference between positions feet. 
$\left.P=0.003 ; \eta_{p}{ }^{2}=0.31\right)$. Sol muscle presented lower RMS values compared to GL (rigth: $P<0.001$; left: $P<0.001, \mathrm{HSD}$ ) and GM (rigth: $P<0.001$; left: $P<0.001, \mathrm{HSD}$ ) as showed at figure 1.

Strength training effect is associated to exercise type, biomechanical muscle activation through load, repetition and sets, as well the training duration and fatigue resistance. The efficacy of weight training has strong relationship to muscle response that is associated to muscular activation with different resistance and contraction length, duration amplitude and limb position. The analysis of electromyography sign is an important methodology to muscle contraction performance of TS due to the importance on daily physical activity as well sports performance. Thus, this research aimed to analyze the EMG responses of TS in the heel-rise exercise performed on leg press machine with different feet positions. The main hypothesis of the present study was to test whether TS EMG signs of muscle conditions, is higher in gastrocnemius muscle compared with soleus muscle, independently of feet position. In addition, we also expected larger participation of gastrocnemius muscle activation compared to soleus, due to the knee position during exercises (extension) $)^{(6)}$.

Our hypothesis was confirmed and our data suggested differences on electromyographic responses on gastrocnemius and soleus, independently of feet position. This data may be explained the different absence of conditions may be related to the joint movement necessary to modify the position of the feet between the variations.

Although it was previously observed that feet position change in the 3 experimental conditions showing that external or lateral rotation (feet pointed out), internal or medial (feet pointed inwards) and its neutral position (feet pointed forward) will impact on the hip position, evidencing that the hip movement in the transverse plane, will change the lower limbs movement, leading to small or no change in the position and movement of knees and ankles ${ }^{(13)}$.

On the other hand, previous study ${ }^{(10)}$ also reported differences between gastrocnemius activation with different plantar flexion position in extension knees exercises, showing that greater medial gastrocnemius activation in relation to the lateral one in the exercise performed with the foot turned outwards and greater activation of the lateral gastrocnemius in relation to the medial in the variation with the foot rotated inwards. It is important to emphasize that in the aforementioned study ${ }^{(10)}$, the exercise was performed in a standing position and not in the leg press, as in the present study.

The EMG activity of lateral gastrocnemius in unipodal squats performance with different feet placements ${ }^{(14)}$ presented similar trend in comparison with our study even the neutral feet, plantar flexion, dorsal flexion, supination and pronation were different from those used in our analysis (standing in external and internal rotation).
In the comparison among muscles, gastrocnemius presented greater activation than soleus, regardless of exercise variation. Baptista et al. ${ }^{(15)}$ observed the gastrocnemius activation presented $40.5 \%$ (lateral) and $31.7 \%$ (medial) higher in RMS value when knee flexion was controlled. Also, knee flexion implied to soleus an increase of $35.6 \%$ in RMS when the knee was extended. It is important to consider that the ankle plantar flexion performed in the leg press machine does not causes knee flexion. These findings were confirmed by Fiebert et al. ${ }^{(16)}$, that demonstrated higher neural action in submaximal contractions (and using different loads) at gastrocnemius, when compared to the soleus.

We consider that the length and angulation of the fascicles may help to explain the results. Considering that longer fascicles, GL has more sarcomeres in series and GM presents in its architecture larger angulations of the fascicles it may be favorable to force and velocity production ${ }^{(17)}$. Also, we may consider that similar muscle fiber composition is similar to $\mathrm{GL}$ and $\mathrm{GM}$, what is not the same to soleus. It seems to be disadvantageous for structural and architectural to force and power response ${ }^{(18)}$.

Thus, the data of the present study lead us to believe that the feet variation position in the heel-rise exercise performed on leg press machine is an ineffective strategy to alter the recruitment of the triceps surae muscle. The main limitations of the present study was the absence of separate analysis of muscle activation between concentric and eccentric phases, as well as non-achievement of maximal isometric voluntary action.

\section{CONCLUSION}

In conclusion, the change in the feet position during the heel-rise exercise performed in the leg press machine does not influence the activation of the triceps surae, and the soleus is less activated than the gastrocnemius in that exercise.

\section{ACKNOWLEDGEMENT}

The authors thank CAPES (Coordenação de Aperfeiçoamento de Pessoal de Nível Superior) fellowships addressed to performed this study. The fund providers had no role in decision to publish and preparation of the paper.

\section{AUTHORS' CONTRIBUTIONS}

Each author contributed individually and significantly to the development of the manuscript. RSP, JBA, RMP and AJS performed the laboratory procedures, as well as recruiting the volunteers, conducting the anthropometric measurements and filling out the questionnaires, and training volunteers. The treatment of the signs obtained and evaluated the data from the statistical analysis was done by FP, MRRP, ALE, CVLST. The revision of the manuscript and contributions to the intellectual concept of the study were provided and the main contributors to the writing of the manuscript by $A C L, A F J, V M R$ and DSB.

\section{CONFLICT OF INTEREST}

All authors declare no potential conflict of interest related to this article. 


\section{AUTHOR DETAILS}

1Translational Laboratory of Physiology, Postgraduate Program in Physical Education and Health Sciences, Universidade São Judas Tadeu, São Paulo, Brazil. ' ${ }^{3}$ ducation Department, Universidade Nove de Julho (UNINOVE), São Paulo, Brazil. ${ }^{4}$ Faculdade de Educação Física, Faculdade Praia Grande (FPG), Praia Grande, Brazil. ${ }^{5}$ Grupo de Estudos da Obesidade. Laboratório Interdisciplinar de Doenças Metabólicas, Universidade Federal de São Paulo, Santos, Brazil. ${ }^{6}$ Postgraduate Program in Applied Biophotonics in Health Sciences, Nove de Julho University (UNINOVE), São Paulo, Brazil. 7 Center for Research in Sports Sciences, Health Sciences and Human Development at the University of Trás-os-Montes \& Alto Douro, Vila Real, Portugal.

\section{REFERENCES}

1. Aronow MS, Diaz-Doran V, Sullivan RJ, Adams DJ. The effect of triceps surae contracture force on plantar foot pressure distribution. Foot Ankle Int. 2006;27:43-52.

2. Dalmau-Pastor M, Fargues-Polo B Jr, Casanova-Martínez D Jr, Vega J, Golanó P. Anatomy of the triceps surae: a pictorial essay.. Foot Ankle Clin. 2014;19:603-635.

3. Magnusson SP, Aagaard P, Rosager S, Dyhre-Poulsen P, Kjaer M. Loaddisplacement properties of the human triceps surae aponeurosis in vivo. J Physiol. 2001;531:277-288.

4. Rosager S, Aagaard P, Dyhre-Poulsen P, Neergaard K, Kjaer M, Magnusson SP. Load-displacement properties of the human triceps surae aponeurosis and tendon in runners and non-runners. Scand J Med Sci Sports. 2002;12:90-98.

5. Bezodis I, Kerwin D, Salo A. Lower-limb mechanics during the support phase of maximum-velocity sprint running. Med Sci Sports Exerc. 2008;40:707-715.

6. Flanagan S, Song JE, Wang MY, Greendale G, Azen S, Salem G. Biomechanics of the heel-raise exercise. J Aging Phys Activ. 2005;13:160-171.

7. Vilaça-Alves J, Guimarães F, Rosa C, Neves EB, Saavedra FJ, Reis VM. Electromyography analysis of the abdominal crunch In stable and unstable surface. Gazzetta Medica Italiana. 2016;175:189-194.
8. Dorel S, Couturier A, Hug F. Intra-session repeatability of lower limb muscles activation pattern during pedaling. J Electromyogr Kinesiol. 2008;18:857-865.

9. Capaday $\mathrm{C}$, Stein RB. Difference in the amplitude of the human soleus $\mathrm{H}$ reflex during walking and running. J Physiol (Land). 1987;392:513-522.

10. Riemann BL, Limbaugh GK, Eitner JD, LeFavi RG. Medial and lateral gastrocnemius activation differences during heel-raise exercise with three different foot positions. J Strength Cond Res. 2011;25:634-639.

11. Freriks B, Hermens HJ. European recommendations for surface electromyography: results of the SENIAM project. Enschede: Roessingh Research and Development; 1999.

12. Cohen J. Statistical power analysis for the behavioral sciences. New York: Academic Press; 1969.

13. Hamill J, Knutzen KM. Biomechanical basis of human movement. 2.ed. Philadelphia: Lippincott Williams and Wilkins; 1995.

14. Ribeiro G, Dionísio VC, Almeida LG. Electromyographic activity during one-legged squatting under different foot positions. Braz J Sports Med. 2007;13:43-46.

15. Baptista MT, Do Nascimento FXM, Nardes LK, Da Matta TT, De Oliveira LF. Influência de posições do joelho no torque e atividade mioelétrica do tríceps sural na flexão plantar isométrica máxima. Rev Bras Educ Fís Esporte. 2014 (inpress).

16. Fiebert I, Spieholz N, Applegate E, Carbone M, Gonzales G, Gorack W. Integrated EMG study of the medial and lateral heads of the gastrocnemius during isometric plantar flexion with varying cuff weights loads. J Back Musculoskel Rehab. 1998;11:19-26.

17. Kawakami Y, Ichinose Y, Fukunaga T. Architectural and functional features of human triceps surae muscles during contraction. J Appl Physiol. 1998;85:398-404.

18. Johnson MA, Polgar J, Weightman D, Appleton D. Data on the distribution of fibre types in thirty-six human muscles: an autopsy study. J Neurol Sci. 1973;18:111-129. 